\title{
A Mechanism of Basal Spacing Reduction in Sodium Smectitic Clay Materials in Contact with DNAPL Wastes
}

\author{
Derya Ayral Cinar ${ }^{1}$, Margarita Otero-Diaz ${ }^{2}$, and Avery H. Demond ${ }^{3 *}$ \\ ${ }^{1,2,3}$ Department of Civil and Environmental Engineering \\ University of Michigan
}

Ann Arbor, Michigan, 48109-2125

USA

\author{
*Corresponding author \\ Rm. 120, EWRE Building \\ 1351 Beal Ave. \\ Ann Arbor, MI, 48109-2125 USA \\ EMAIL: averyd@umich.edu \\ PHONE: (734) 763-3708
}


Abstract

There has been concern regarding the possible attack of clays in aquitards, slurry walls

3 and landfill liners by dense nonaqueous phase liquid (DNAPL) wastes, resulting in cracking.

4 Despite the fact that a reduction in basal spacing in sodium smectitic clay materials has been

5 linked to cracking, no plausible mechanism by which this reduction occurs in contact with waste

6 DNAPLs has been formulated. To elucidate a mechanism, screening studies were conducted that

7 showed that the combination of an anionic surfactant (AOT), a nonionic surfactant (TritonX-

8 100) and a chlorinated solvent, tetrachloroethylene (PCE), could replicate the basal spacing

9 reduction and cracking behavior of water-saturated bentonite caused by two waste DNAPLs

10 obtained from the field. FTIR measurements of this system showed a displacement of the H-O-

$11 \mathrm{H}$ bending band of water symptomatic of desiccation. Sorption measurements showed that the

12 uptake of AOT by bentonite increased eight fold in the presence of TritonX-100 and PCE. The

13 evidence presented here supports a mechanism of syneresis, involving the extraction of water

14 from the interlayer space of the clay through the synergistic sorption of a nonionic and anionic

15 surfactant mixture. It is speculated that the solvation of water in reverse micellar aggregates is

16 the process driving the syneresis.

18 Keywords: clay; cracking; syneresis; DNAPL; surfactant, hazardous waste 


\section{Introduction}

Tetrachloroethylene (PCE) and trichloroethylene (TCE) are chlorinated dense nonaqueous phase liquids (DNAPLs) that are commonly identified at hazardous waste sites

22 (Oolman et al., 1995, Dwarakanath et al., 2002; Dou et al., 2008; Parker et al., 2008). The migration of DNAPLs in the subsurface may be constrained by the presence of clay lenses and aquitards, or by the installation of engineered slurry walls. The low permeability of these materials results from the presence of clay; in the case of slurry walls, the clay is bentonite, or primarily sodium montmorillonite. However, there has been concern that bentonite may be attacked by chlorinated solvents (Abdul et al., 1990; McCalou and Huling, 1999). Studies investigating this phenomenon have used changes in hydraulic conductivity as their measure of the degree of alteration, citing that the permeation of the clay with a chlorinated solvent resulted in an increase of up to two orders of magnitude. The explanation given was that the solvent displaced water, causing double-layer compression because of the low dielectric constant of the solvent. The shrinkage resulted in the formation of a network of cracks, which facilitated the flow of the solvent through the clay. Similar behavior was not observed in cases where water saturated with the solvent was used as the permeating fluid. clays has surfaced in the context of mass storage in clay lenses and aquitards. Due to the longterm contact between DNAPLs in the subsurface and these geologic strata, the latter can accumulate a significant mass of contamination over time, essentially becoming contaminant storage zones (Sale et al., 2008; Stroo et al., 2012). Field work has documented high concentrations of TCE and PCE in clay lenses and aquitards at waste sites (Ball et al., 1997; Parker et al., 2008). Once the original source is removed, these zones slowly release the accumulated contaminants back into the surrounding groundwater, significantly extending remediation times (Chapman and Parker, 2005).

It has been speculated that interactions between DNAPLs and the clay minerals in these layers may play a role in the mass accumulation (Matthieu et al., 2013; Ayral et al., 2014). One possible mechanism by which mass accumulation due to clay-waste interactions may occur is through cracking. If contact with DNAPLs causes a contraction in the basal spacing leading to cracking, the cracks may fill with DNAPL in a manner similar to that hypothesized for naturallyoccurring fractures (Kueper and McWhorter, 1991). However, to substantiate such a hypothesis, 
a clearer mechanistic understanding of crack formation due to contact with DNAPLs is necessary. In the studies showing an increase in hydraulic conductivity of bentonite exposed to neat chlorinated solvents, the clays were subjected to high hydraulic gradients, and it is unclear if the bentonite would have been permeated otherwise, given that water-saturated smectites are negatively-charged (Meunier, 2005), hydrophilic (van Oss and Giese, 1995) and have high entry pressures due to the small size of their pores (Marshall et al., 1996). Furthermore, Ayral et al. (2014) found that passive contact of up to 319 days with laboratory-grade PCE and TCE did not cause cracking, and the few reported measurements of basal spacing of water-saturated smectitic clays in contact with chlorinated solvents are similar to those measured with water, suggesting that these solvents are unable to penetrate into the interlayer space of these materials when they are saturated with water (Griffin et al., 1984; Ayral et al., 2014).

Although the passive contact of smectitic clays with neat chlorinated solvents did not have an impact, contact with two chlorinated DNAPLs recovered from the field reduced the basal spacing of bentonite from about $19 \AA$ to $15 \AA$ and caused the formation of cracks of up to 1 $\mathrm{mm}$ in aperture in a time frame of weeks (Ayral et al., 2014). While the predominant organic component in a chlorinated DNAPL may be PCE or TCE, DNAPLs contain a range of solutes, added to enhance their performance (Jackson and Dwarakanath, 1999; Linn and Stupak, 2009), obtained through their use (Jackson and Dwarakanath, 1999) or acquired during their residence in the subsurface (Harrold et al., 2003). Additives to degreasing and drycleaning solvents include alcohols, aliphatic ketones, carboxylic esters, glycol ethers, and surfactants (Earnest et al., 1997; Jackson and Dwarakanath, 1999; Linn and Stupak, 2009); organic components identified in actual PCE and TCE field wastes include carboxylic esters, fuel hydrocarbons and phthalates (Dou et al., 2008), and nonionic and anionic surfactants (Hsu, 2005). In fact, Jackson and Dwarakanath (1999) point out that any chlorinated solvent in the subsurface will contain solutes that may significantly influence its behavior.

One hypothesis of how the basal spacing reduction and the ensuing cracking may occur in smectitic clays is that water-soluble organic compounds present in the waste dissolve in the water present in the interlayer spacing of the clay, enhancing the dissolution of the chlorinated solvent within the clay. Matthieu et al. (2013) cite evidence that the intercalation of TCE in sodium montmorillonite is possible. Furthermore, the ability of a cosolvent to increase the solubility of hydrophobic organic compounds is well documented (Schwarzenbach et al., 2003). 
81 The solubility of PCE can be increased by orders of magnitude by the presence of an alcohol, for example (Imhoff et al., 1995; Ladaa et al., 2001). Whether a mixture comprising a chlorinated solvent and an alcohol would result in a reduction of the basal spacing, however, is unclear. Contact of water-saturated bentonite with an aqueous solution of TCE at the solubility limit yielded a basal spacing similar to that in contact with water (18.2 $\AA$ [Matthieu et al., 2013] vs. 18.0 -18.2 Å [Brown and Thomas, 1987; Li et al., 1996]). But, aqueous mixtures containing on the order of $10 \%$ ethanol have been shown to expand the basal spacing beyond that in contact with water alone (Griffin et al., 1984; Brown and Thomas, 1987).

Other hypotheses may be based on surfactant content. An explanation centered on altered wettability by surfactants is not supported, however, as the DNAPL systems examined in Ayral et al. (2104) were water-wet (Hsu, 2005), similar to the situation with the DNAPL waste from the Savannah River site (Dou et al., 2008). Alternatively, studies of organobentonites show that cationic surfactants can sorb in the interlayer space, increasing the basal spacing (e.g., Lee et al., 2004; Hu et al., 2013) and the sorption of hydrophobic organic chemicals (e.g., Boyd et al., 1988; Zhu et al., 1998). However, the increased sorption of hydrophobic chemicals was related to the sorption of cationic surfactants, and Hsu (2005) identified nonionic and anionic surfactants in the DNAPL waste samples. Work examining the sorption of nonionic surfactants documents a lesser expansion of the clay structure (Shen, 2001; Deng et al., 2003) and the intercalation of this type of surfactant did not seem to enhance the sorption of hydrophobic organic compounds such as toluene or cyclohexane (Deng et al., 2003). In fact, Deng et al. (2003) speculated that nonionic surfactants were not fully penetrating the interlayer space and that the sorption that did occur was primarily confined to the interlayer margins. Similarly, Sanchez-Martin et al. (2008) concluded that the minimal sorption of sodium dodecylsulfate (SDS), an anionic surfactant, to montmorillonite, probably also occurred on the margins, due to the lack of change in the basal spacing.

In the context of a DNAPL waste, possible synergistic interactions must also be considered (Hsu and Demond, 2007). For example, the sorption of an anionic surfactant onto kaolinite from a mixture of anionic-nonionic surfactants could be greater than that in the presence of the anionic surfactant itself (Xu et al., 1991). Hydrophobic chain-chain interactions of the surfactants and the reduction of the repulsive force of the negatively-charged head of the anionic surfactant by the nonionic surfactant were offered as a possible explanation for such 
112 synergy (Xu et al., 1991; Zhang and Somasundaran, 2006). It should be noted that all of these

113 studies examined sorption from aqueous solutions; comparable sorption studies from nonpolar

114 solvents are not available. However, studies of surfactant behavior in nonpolar solvents show

115 that surfactants can form mixed reverse micelles (Fendler, 1976; Moulik, 1996), based on

116 interactions between the hydrophilic moieties of the anionic and nonionic surfactants.

117 Furthermore, these reverse micelles solubilize water in their core (Silber et al., 1999), a

118 phenomenon that is exploited in the dry cleaning industry for the removal of water-based stains

119 (Linn and Stupak, 2009).

120

121

122

123

124

125

126

127

128

129

130

131

132

133

134

135

136

137

138

139

140

141

Although solutes present in the DNAPL wastes may play a critical role in the contraction of the basal spacing of bentonite, and consequently, possibly in the mass accumulation of contaminants by aquitards or in the deterioration of slurry walls and landfill liners, the mechanism by which this occurs is unknown. Previous studies evaluating the possible degradation of bentonite in contact with chlorinated solvents hypothesized that the DNAPL enters the clay, causing double-layer compression and subsequent cracking, without offering supporting evidence. But, how the DNAPL actually enters the clay, or whether it even does, is a matter of speculation. Thus, the objective of this study was to delineate a plausible mechanism by which chlorinated DNAPL wastes can cause a contraction of the structure of water-saturated bentonite.

\section{Screening Experiments}

A preliminary step in obtaining this objective was to perform two sets of screening experiments to ascertain which components of the waste were the causative agents of the reduction in basal spacing. Two DNAPLs, one a PCE-based DNAPL obtained from a dry cleaner (Ann Arbor, MI), and the other a TCE-based DNAPL obtained from Operable Unit 2 at Hill Air Force Base (UT), both of which Ayral et al. (2014) found to reduce the basal spacing of sodium smectites and cause cracking, served as the representative wastes. Both wastes had contact angles on quartz in the presence of water at neutral $\mathrm{pH}$ of approximately $30^{\circ}$. The interfacial tension of both waste was about 2-3 dyn/cm, attributed to the presence of nonionic and anionic surfactants (Hsu, 2005). To ascertain whether water-soluble organic components in the DNAPL wastes were enhancing the aqueous solubility of the chlorinated solvent, the wastes were contacted with water at a waste-to-water volume ratio of 4:1 for 56 days. After this period 
142 of equilibration, the aqueous phase was extracted with toluene, which was then analyzed for

143 concentrations of PCE and TCE by gas chromatography. The PCE concentration in the aqueous

144 phase contacted with the PCE-based waste was measured to be $9500 \mathrm{mg} / \mathrm{L}$, with no measurable

145 concentrations of TCE. The concentrations in the aqueous phase contacted with the degreasing

146 TCE waste were $125 \mathrm{mg} / \mathrm{L}$ and $500 \mathrm{mg} / \mathrm{L}$, of PCE and TCE respectively. The aqueous

147 solubilities of PCE and TCE at $25^{\circ} \mathrm{C}$ are $150 \mathrm{mg} / \mathrm{L}$ and $1370 \mathrm{mg} / \mathrm{L}$, respectively (Riddick et al.,

148 1986). Thus, the components in the PCE-based waste increased the aqueous phase concentration

149 of PCE significantly, but the components in the TCE-based waste reduced the TCE concentration

150 below solubility. Since both the PCE and TCE wastes caused similar basal spacing contraction

151 and cracking (Ayral et al., 2014), but the aqueous solubility increased only in the case of the PCE

152 waste, an enhancement of the solubility of the chlorinated compounds in the interlayer water was

153 eliminated as a likely mechanism.

154 The second set of screening experiments involved setting up a suite of 18 20-mL glass

155 vials. One gram of commercial Wyoming Na-bentonite (Southwestern Materials, Austin, TX)

156 was put into each vial and contacted with $10 \mathrm{~mL}$ of a $0.005 \mathrm{M} \mathrm{CaSO}_{4}$ solution to saturate it.

157 After two weeks, the surficial excess water was removed. Then both aqueous and PCE solutions

158 were made up containing various combinations of organic solutes hypothesized to be present in

159 dry cleaner and degreasing wastes, at concentrations given in Table 1. Then, $15 \mathrm{~mL}$ of one of

160 these solutions was added on top of the clay. The vials were capped, stored at room temperature

161 and observed over time. No cracking was observed for over 75 days in the vials containing an

162 individual or a combination of the lower-molecular weight organic solutes dissolved in PCE.

163 Moreover, vials containing a single surfactant dissolved in PCE did not show evidence of rapid

164 cracking. Yet, cracking occurred in less than two weeks in the vials containing the anionic and

165 nonionic surfactants and PCE. Therefore, it was concluded that a surfactant combination was

166 vital to the cracking process. As the same surfactants dissolved in water did not cause cracking,

167 the implication was that the chlorinated solvent was also an indispensable constituent.

\section{Experimental Methods}

Once the minimum necessary system components were determined, x-ray diffraction

170 (XRD), Fourier transform infrared (FTIR) spectroscopy and sorption measurements were made

171 to gain additional insight into the mechanism. Water-saturated bentonite samples were prepared 
Table 1. Concentrations of Compounds in Screening Experiments

\begin{tabular}{cccc}
\hline Chemical group & Compound & Chemical Formula & Concentration \\
\hline Alcohol & Propanol & $\mathrm{C}_{3} \mathrm{H}_{8} \mathrm{O}$ & $50 \mathrm{mg} / \mathrm{L}$ \\
\hline \multirow{2}{*}{ Aliphatic ketone } & Acetone & $\mathrm{C}_{3} \mathrm{H}_{6} \mathrm{O}$ & $10 \mathrm{mg} / \mathrm{L}$ \\
& Methyl isobutyl ketone & $\mathrm{C}_{6} \mathrm{H}_{12} \mathrm{O}$ & $2550 \mathrm{mg} / \mathrm{L}$ \\
\hline \multirow{2}{*}{ Aliphatic carboxylic ester } & Amyl acetate & $\mathrm{C}_{7} \mathrm{H}_{14} \mathrm{O}_{2}$ & $525 \mathrm{mg} / \mathrm{L}$ \\
& n-Butyl acetate & $\mathrm{C}_{6} \mathrm{H}_{12} \mathrm{O}_{2}$ & $528 \mathrm{mg} / \mathrm{L}$ \\
\hline Glycol ether & 2-Butoxyethanol & $\mathrm{C}_{6} \mathrm{H}_{14} \mathrm{O}_{2}$ & $8992 \mathrm{mg} / \mathrm{L}$ \\
\hline \multirow{2}{*}{ Nonionic surfactant } & TritonX-100 & $\mathrm{C}_{14} \mathrm{H}_{22} \mathrm{O}\left(\mathrm{C}_{2} \mathrm{H}_{4} \mathrm{O}\right)_{\mathrm{n}}$ & $3.3 \mathrm{mM}$ \\
& $\mathrm{C}_{12} \mathrm{E}_{6}{ }^{\mathbb{T}}$ & $(\mathrm{n}=9-10)$ & $6.7 \mathrm{mM}$ \\
\hline Anionic surfactant & $\mathrm{AOT}$ & $\mathrm{C}_{24} \mathrm{H}_{50} \mathrm{O}_{7}$ & $3.3 \mathrm{mM}$
\end{tabular}

173

174

175

176

177

178

179

180

181

182

183

184

185

186

187

188

189

190

191

192

193

194

195

196

197

Aqueous solutions were made up using water with a resistivity of $18.3 \mathrm{M} \Omega \cdot \mathrm{cm}$ made by passing deionized distilled water through a series of four cartridges (Milli-Q, EMD Millipore) to remove additional impurities. PCE solutions were made up using HPLC grade PCE $(>99.9 \%$, Sigma Aldrich). All other chemicals were laboratory grade from Fisher Scientific, unless noted. ${ }^{\dagger}$ ICN Biomedicals; "Sigma Aldrich.

as in the screening experiments by adding $10 \mathrm{~mL}$ of $0.005 \mathrm{M} \mathrm{CaSO}_{4}$ to one gram of bentonite in glass vials. After two weeks, the excess water was removed and the clay was mixed for homogeneity. Two grams of the clay were then transferred into a 30-mL centrifuge tube and 20 $\mathrm{mL}$ of a solution containing combinations of TritonX-100, AOT and $\mathrm{C}_{12} \mathrm{E}_{6}$ as solutes, dissolved in either water or PCE, were added into the tubes. The first set of tubes was rotated for four days followed by passive contact of at least one week; the second set of tubes was rotated for three weeks. The clay-solution mixture was centrifuged and the solid phase was analyzed using an Xray diffractometer (Rigaku, The Woodlands, TX) equipped with a rotary anode source (CU) with a $12 \mathrm{~kW}$ X-ray generator, a graphite monochromator and two wide angle horizontal goniometers $\left(2^{\circ}-138^{\circ}\right)$. As the samples containing chlorinated solvent had diffuse peaks, they were analyzed using a step size of $0.04^{\circ}$ and a count time of $20 \mathrm{sec}$ over the range of $4-8^{\circ} 2 \theta$. JADE (Version 10; Materials Data, Livermore, CA) was used to analyze the data. A background curve was fitted automatically and, after determining the portion of the profile attributable to the background, the PseudoVoigt profile function was used to fit the profile and determine the location of the peaks.

Samples were also analyzed using FTIR spectroscopy (Spectrum BX, Perkin Elmer, Boston, MA) with a MIRacle attenuated total reflectance (ATR) accessory. First, the background was scanned in a range of $650 \mathrm{~cm}^{-1}$ to $4000 \mathrm{~cm}^{-1}$ at a resolution of $4 \mathrm{~cm}^{-1}$. Then, the sample was placed on the ATR crystal, and data was collected in the transmission mode. The 
spectra were corrected using the background scan and the peaks on each spectrum greater than the threshold (5\% of transmission) were labeled. The bentonite clay samples were rotated for four days and then were left sitting for almost six months. For comparison, scans were also made for dry and wet clay, clay in contact with aqueous solutions of surfactants, and for solvents and solutions in the absence of clay.

For the sorption measurements, vials were made up containing one gram of Wyoming Na-montmorillonite clay (SWy-2; Clay Minerals Society, Chantilly, VA) that was contacted with $10 \mathrm{~mL}$ of $0.005 \mathrm{M} \mathrm{CaSO}_{4}$ solution for two weeks. Similar to the protocol in the basal spacing measurements, excess surficial water was removed from the surface and the clay was mixed. Then, two or four grams of the clay were transferred to a $30-\mathrm{mL}$ centrifuge tube. Surfactant solutions containing the same concentrations as in the screening experiments (Table 1) were prepared in $50 \mathrm{~mL}$ volumetric flasks, and in the case of the surfactant solutions prepared in PCE, $0.06 \mathrm{~mL}{ }^{13} \mathrm{C}$-labeled TCE (>98\%; Cambridge Isotope Laboratory, MA) was also injected into the volumetric flasks. $20 \mathrm{~mL}$ of the surfactant solutions were then added to the centrifuge tubes. Following one week of rotation, the tubes were centrifuged. The supernatant phase was sampled to determine the concentration of surfactant and ${ }^{13} \mathrm{C}$-labeled TCE left in the solution, from which the degree of sorption was calculated by mass balance.

The surfactant concentrations in both the aqueous phase and PCE were analyzed using high-performance liquid chromatographs (HPLC) (Hewlett Packard), equipped with evaporative light scattering detectors (ELSD) (Sedere Sedex), as detailed in Ayral (2015). The concentration of ${ }^{13} \mathrm{C}$-labeled TCE in PCE was measured using a gas chromatograph (Hewlett-Packard 5890) equipped with a mass selective detector (Hewlett-Packard 5972) to differentiate between bulk TCE and ${ }^{13} \mathrm{C}$-labeled TCE, based on a shift of the base peaks. Ions at $\mathrm{m} / \mathrm{z}$ values of 131,133 , and 135 were extracted and used to determine the concentration of ${ }^{13} \mathrm{C}$-labeled TCE.

\section{Results and Discussion}

The screening experiments suggested that a mixture of anionic and nonionic surfactants dissolved in a chlorinated solvent was necessary in order to observe a magnitude and rate of cracking comparable to the DNAPL wastes. The XRD results (Table 2) show that an aqueous solution of TritonX-100 reduced the basal spacing of water-saturated bentonite from $19.0 \AA$ to 
17.5 $\AA$; the combination of TritonX-100 and AOT had the similar impact. In contrast, contact with an aqueous solution of all three surfactants (TritonX-100, AOT, and $\mathrm{C}_{12} \mathrm{E}_{6}$ ) caused the basal

Table 2. Basal Spacing of Bentonite (Initially Saturated with $0.005 \mathrm{M} \mathrm{CaSO}_{4}$ ) in Contact with Various Surfactant Solutions

\begin{tabular}{|c|c|c|c|c|}
\hline \multirow[b]{2}{*}{ Surfactant } & \multicolumn{2}{|c|}{$\begin{array}{c}\text { Basal spacing }(\AA) \\
\text { (4 days of rotation and } \\
\text { one week passive } \\
\text { contact) }\end{array}$} & \multicolumn{2}{|c|}{$\begin{array}{c}\text { Basal spacing }(\AA) \\
\text { (3 weeks of rotation) }\end{array}$} \\
\hline & $\begin{array}{l}\text { Dissolved } \\
\text { in water }\end{array}$ & $\begin{array}{l}\text { Dissolved } \\
\text { in PCE }\end{array}$ & $\begin{array}{l}\text { Dissolved } \\
\text { in water }\end{array}$ & $\begin{array}{l}\text { Dissolved } \\
\text { in PCE }\end{array}$ \\
\hline No surfactant & 19.0 & 19.0 & NM & $\mathrm{NM}$ \\
\hline TritonX-100 (3.3 mM) & 17.5 & 17.5 & 17.4 & 15.6 \\
\hline $\begin{array}{c}\text { TritonX-100 (3.3 mM) and AOT (3.3 } \\
\mathrm{mM})\end{array}$ & 17.9 & 15.4 & 17.1 & 12.7 \\
\hline $\begin{array}{c}\text { TritonX-100 (3.3 mM) and } \mathrm{C}_{12} \mathrm{E}_{6}(6.7 \\
\mathrm{mM})\end{array}$ & NM & 18.8 & NM & NM \\
\hline $\begin{array}{c}\text { TritonX-100 (3.3 mM), AOT }(3.3 \mathrm{mM}) \\
\text { and } \mathrm{C}_{12} \mathrm{E}_{6}(6.7 \mathrm{mM})\end{array}$ & 20.2 & 15.8 & NM & NM \\
\hline
\end{tabular}

NM: not measured.

spacing to increase to $20.2 \AA$. But, water-saturated bentonite in contact with the same surfactant mixture in PCE showed a reduction in the basal spacing to $15.8 \AA$, similar to that observed when water-saturated bentonite was contacted with DNAPL waste. Similarly, the basal spacing of water-saturated bentonite in contact with a PCE solution of TritonX-100 and AOT decreased to 15.4 $\AA$, suggesting that the second nonionic surfactant $\mathrm{C}_{12} \mathrm{E}_{6}$ was not necessary. The basal spacing of water-saturated bentonite in contact with PCE containing these surfactants decreased even further to $12.7 \AA$ after three weeks of rotation, to almost air-dry values. These results showed that the minimum mixture needed to reproduce the basal spacing measurements reported in Ayral et al. (2014) for DNAPL wastes was a combination of TritonX-100, AOT, and PCE.

Figure 1 shows the XRD pattern of water-saturated bentonite contacted with the mixture of AOT and TritonX-100 dissolved in PCE. The average basal spacing is $15.4 \AA$, but in fact, the pattern appears to be comprised of three overlapping peaks: one peak corresponding to a basal spacing of $18.9 \AA$ ( $25 \%$ of the total area as calculated by the profile fitting software), another one to $15.5 \AA$ ( $50 \%$ of the total area), and a third to $11.5 \AA$ ( $25 \%$ of the total area). These values 
suggest that about $25 \%$ of the interlayer space was still fully hydrated, $50 \%$ of the interlayer space was partially hydrated and $25 \%$ of the interlayer space of bentonite was dehydrated up to

250

251

252

253 254

255

256

257

258

259

260

261

262

263

264

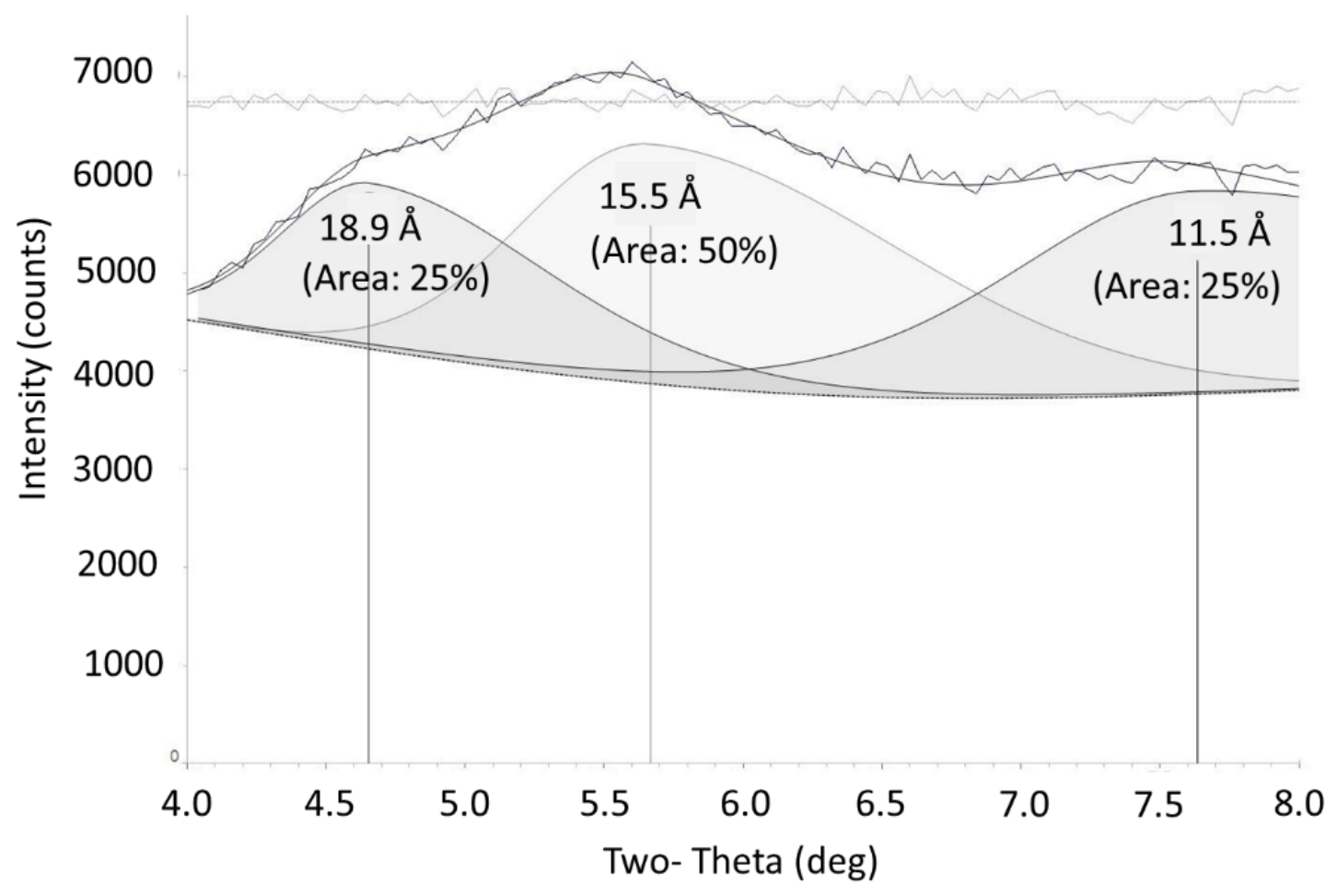

\section{Figure 1. XRD pattern of water-saturated bentonite contacted with PCE containing 3.3 $\mathrm{mM}$ AOT and $3.3 \mathrm{mM}$ TritonX-100 for one week following four davs of rotation.}

air-dry conditions, as $11.5 \AA$ is the basal spacing of air-dry bentonite (Brown and Brindley, 1980; Moore and Reynolds, 1989).

Figure 2 summarizes the FTIR results. The H-O-H bending band of water (Madejova and Komadel, 2001) is located at $1652 \mathrm{~cm}^{-1}$ for air-dry clay and at $1636 \mathrm{~cm}^{-1}$ for water-saturated bentonite. This figure shows that six months of contact with TritonX-100 dissolved in PCE did not change the location of the $\mathrm{H}-\mathrm{O}-\mathrm{H}$ bending band relative to that for water-saturated clay, suggesting that the interlayer water was not impacted by the presence of the nonionic surfactant and chlorinated solvent. This observation agrees with the results of the screening experiments and basal spacing measurements since neither cracking nor a reduction in the basal spacing was observed for this system. However, contact with a mixture of TritonX-100, AOT and PCE caused the wavenumber of the $\mathrm{H}-\mathrm{O}-\mathrm{H}$ bending band to shift to $1652 \mathrm{~cm}^{-1}$, similar to the location for air-dry clay. This shift in wavenumber towards that for air-dry clay suggests a displacement 
of water molecules from the interlayer space, similar to the process of desiccation (Russell and Farmer, 1964; Johnston et al., 1992). Although evidence was found suggesting an interruption of the hydration layers in clay, surfactant specific bands were not detected. In previous studies

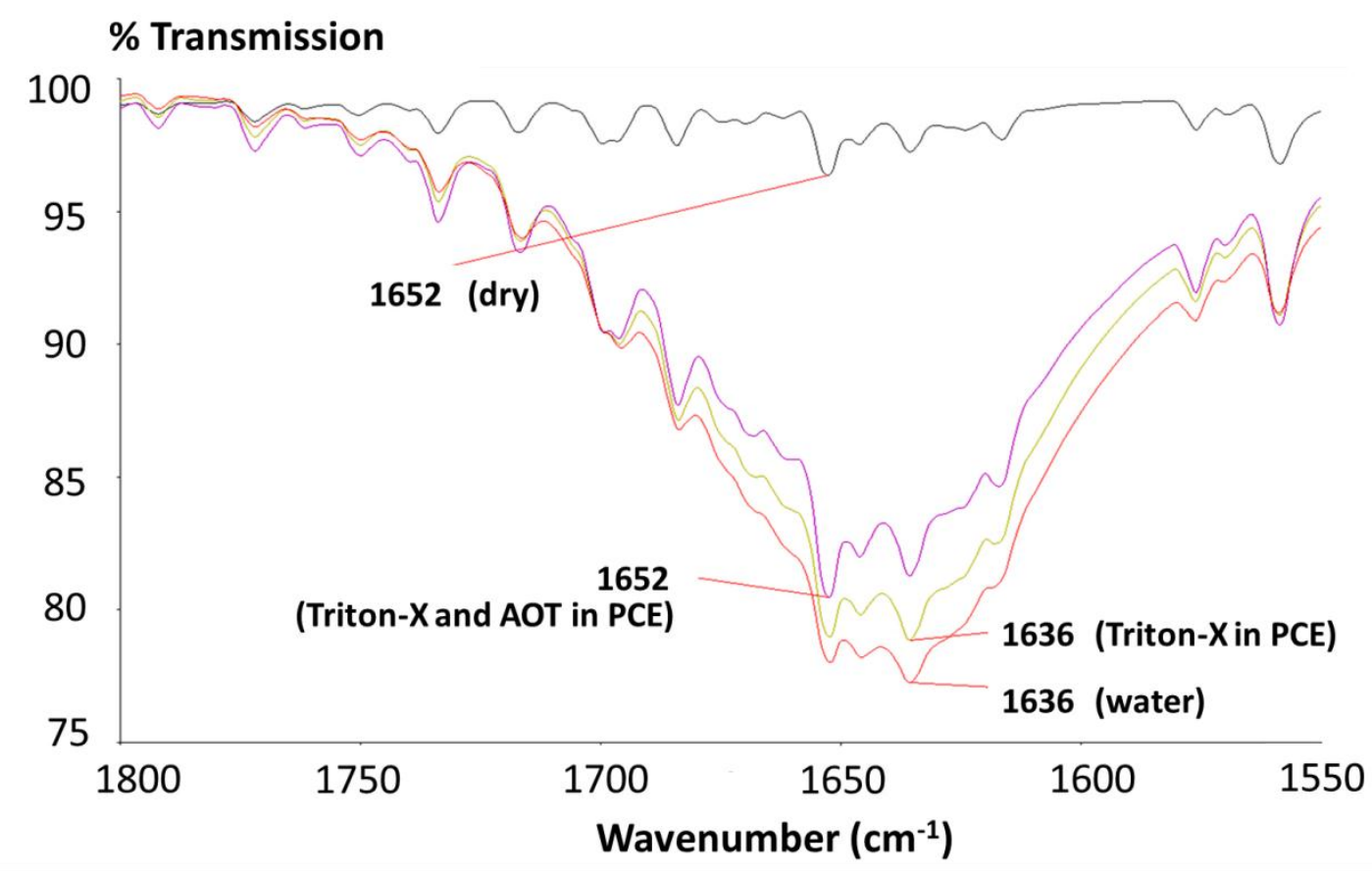

Figure 2. FTIR spectra of water-saturated bentonite in contact with different fluids compared to the spectrum of air-dry bentonite. Wavenumbers indicated correspond to the $\mathrm{H}-\mathrm{O}-\mathrm{H}$ bending band of water.

using FTIR to examine surfactants in clays, the clays were exposed to high concentration surfactant solutions and then dried. Here, the clays were exposed to relatively dilute concentrations of surfactants and were analyzed wet. Because of these differences in measurement conditions, surfactant specific bands, such as those reported by Del Hoyo et al. (2008), could not be observed.

Figure 3 summarizes the results from the sorption experiments conducted in both water and PCE. Figure 3 a shows that that $65 \%$ of TritonX-100 was sorbed from an aqueous solution of TritonX-100, whereas the degree of sorption of TritonX-100 decreased slightly to $57 \%$ if the aqueous solution contained both TritonX-100 and AOT. On the other hand, the degree of AOT sorption increased from $7 \%$ to $14 \%$ if AOT and TritonX-100 were present in the aqueous solution together. Thus, the presence of TritonX-100 increased the sorption of AOT two fold, giving a sorbed molar ratio of TritonX-100:AOT of around 5:1 from the aqueous solution. 

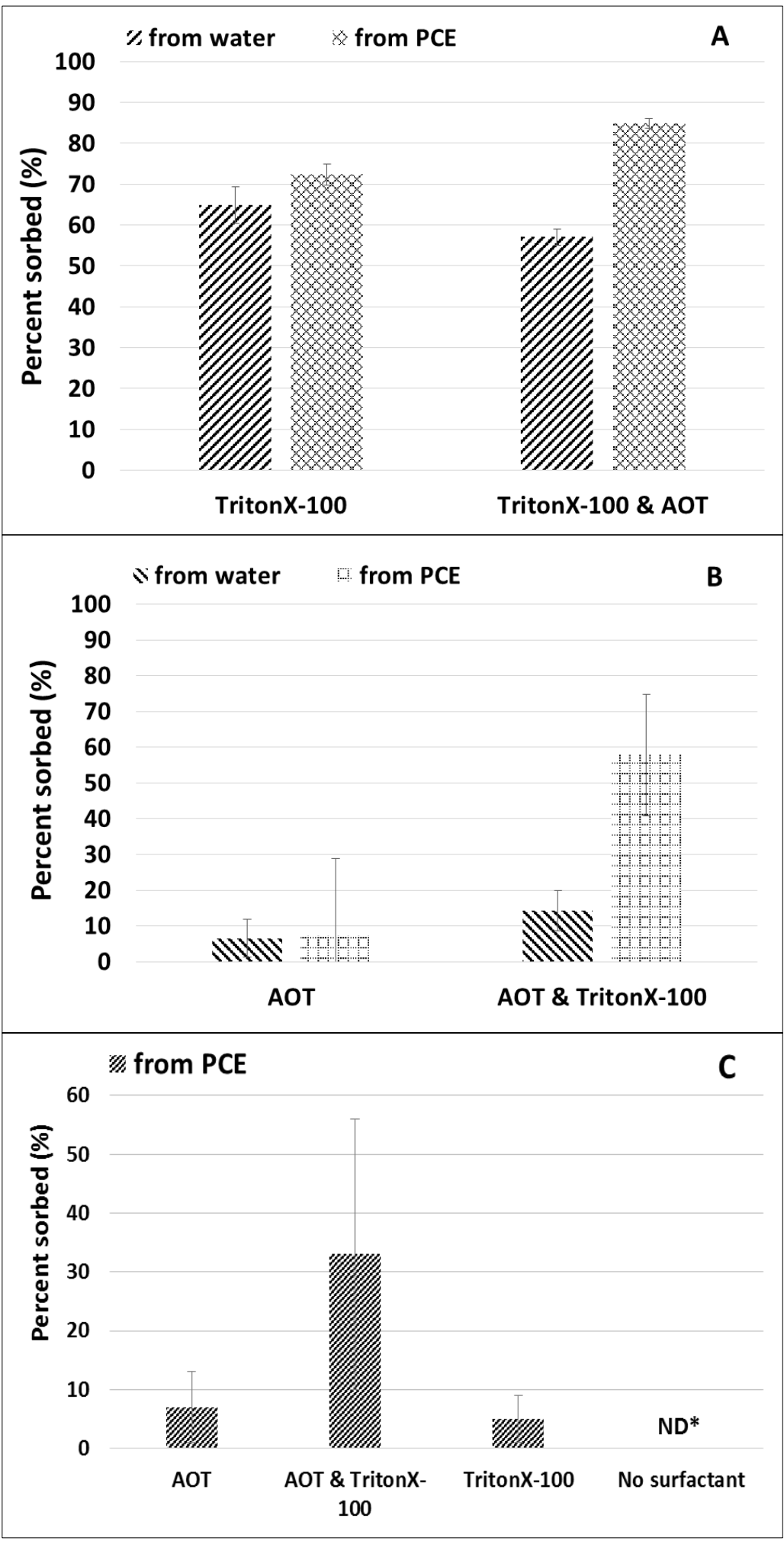

Figure 3. Percent sorption of solutes from aqueous and PCE solutions onto Namontmorillonite. A: TritonX-100; B: AOT; C: ${ }^{13}$ C-labeled TCE. ND = below detection. 
Similar experiments were conducted with the surfactants dissolved in PCE. The results, presented in Figure 3b, showed that the degree of TritonX-100 sorption increased slightly, from $72 \%$ to $85 \%$, in the presence of AOT. On the other hand, the degree of AOT sorption increased almost eight fold, from $7 \%$ to $58 \%$, in the presence of TritonX-100. In the case of sorption from PCE, the sorbed molar ratio of TritonX-100:AOT was 3:2. Although AOT is an anionic surfactant, its sorption increased considerably through its association with a chlorinated solvent and a nonionic surfactant. Measurements of the sorption of ${ }^{13} \mathrm{C}$-labeled TCE (Figure $3 \mathrm{c}$ ) show that, in the absence of surfactant, the degree of sorption was nondetectable. In the presence of surfactants, the sorption of TCE is higher, but the increase is considerably less than that observed in the case of AOT. Thus, the fundamental difference noted in the presence of the chlorinated solvent is increased sorption, in particular, that of the anionic surfactant AOT.

The sorption results suggested that there was increased sorption in the presence of PCE, with about $85 \%$ of the nonionic surfactant, TritonX-100, and nearly $60 \%$ of the anionic surfactant, AOT, sorbed onto water-saturated Na-montmorillonite, giving a sorption ratio of 3:2. The location and orientation of the surfactant molecules relative to the clay surface of the interlayer space will depend, in part, on the size of the molecules (Table 3). The basal spacing for sodium montmorillonite is the sum of the tetrahedral-octahedral-tetrahedral (TOT) layer and the interlayer space. The TOT structure of smectite clay minerals has a thickness of around 9.6 $\AA$ (Moore and Reynolds, 1997); thus, the interlayer space has a thickness of around $5.5 \AA$, if the basal spacing is $15 \AA$. TritonX-100 can conceivably penetrate and sorb in the water-filled interlayer space since it is nonionic, miscible in water, and has a width of about $4 \AA$ (based on the diameter of a phenyl group). But, due to its length, TritonX-100 probably lies essentially parallel to the interlayer surfaces, as also hypothesized by Deng et al. (2003). Conversely, AOT is less likely to enter into the interlayer space because it has a negatively-charged head, whose diameter is $5 \AA$. AOT's enhanced sorption in the presence of TritonX-100 suggests that AOT interacts with TritonX-100, most likely through their hydrophilic moieties because of the nonpolar nature of the surrounding solvent (Moulik, 1996). Furthermore, if there is water present on the exterior surfaces, this may contribute to the association of AOT with the surface, as AOT shows preferential partitioning into water over PCE $\left(\mathrm{K}_{\mathrm{PCE} \text {,water }}=0.001\right)(\mathrm{Hsu}, 2005)$. Assuming a 3:2 ratio of sorption, that interactions between TritonX-100 and AOT occur through 
344 their hydrophilic moieties, that TritonX-100 can penetrate into the interlayer space, but AOT

345 cannot, a schematic can be constructed (Figure 4).

346 With the sorption of TritonX-100, some of the water molecules at the margins of the

347 interlayer space of the clay particle may be displaced. In addition to this displacement of water

Table 3. Dimensions of Molecules in Model DNAPL Waste System

\begin{tabular}{c|c|c}
\hline Head diameter of AOT & $5.0 \AA$ & Moulik and Mukherjee, 1996 \\
\hline Tail length of AOT & $12.6 \AA$ & Moulik and Mukherjee, 1996 \\
\hline Width of TritonX-100 & $4.2 \AA$ & Zhang et al., 2005 \\
\hline Length of TritonX-100 & $48 \AA$ & Paradies, 1980 \\
\hline Width of PCE molecule & $3.6 \AA$ & Zhou, 1994 \\
\hline Width of water molecule & $2.8 \AA$ & Cheng et al., 2001 \\
\hline
\end{tabular}

$349{ }^{\S}$ Taken as the diameter of the phenyl group. ${ }^{\text {IT Taken as effective hydrodynamic radius of the micelle at }}$ $35020^{\circ} \mathrm{C}$.

351

352 


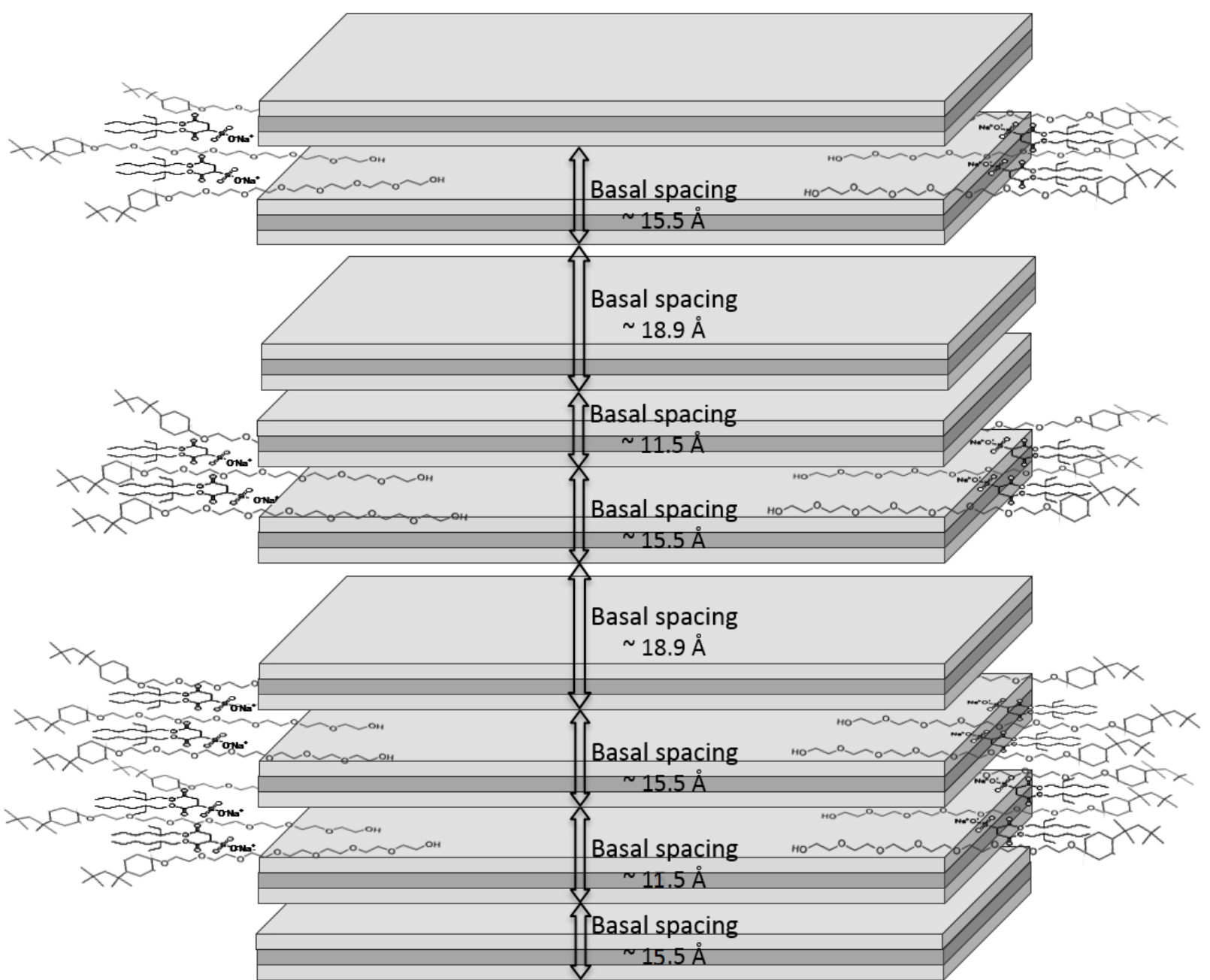

Figure 4. Schematic representation of possible arrangement of AOT and TritonX-100 in PCE in contact with water-saturated bentonite.

by TritonX-100, it is speculated that the association of the AOT molecules in close proximity with the interlayer space stimulates further reductions in the quantity of the interlayer water. AOT is known to spontaneously form thermodynamically stable premicellar aggregates and reverse micelles in nonpolar solvents, with water being an essential component in their formation. These aggregates can solubilize substantial amounts of water, reaching molar ratios of water to micellized surfactant of up to 40-60, depending on the temperature and the nature of the nonpolar solvent (Silber et al., 1999). Thus, AOT's association with TritonX-100 may facilitate its attraction of water from the interlayer structure to fulfill its need for aqueous solvation. As the displacement of water molecules from the interlayer space is not uniform, 
there is a range of basal spacings; this is reflected in the XRD pattern (Figure 1) as a very broad peak which actually represents overlapping peaks, with each peak indicating interlayer space with different thicknesses.

The explanation of how bentonite cracks in contact with organic solvents that has been put forth previously is one of intercalation, involving the entrance of the chlorinated compound into the interlayer space. The evidence presented here supports a mechanism primarily of syneresis, involving the extraction of water from the interlayer space by the synergistic sorption of nonionic and anionic surfactants. Burst (1965) documented the cracking of clay layers containing as little as $10 \%$ bentonite due to syneresis, driven by high salinity levels in the surrounding aqueous solution. The evidence presented in this study suggests that cracking may be similarly precipitated by the solvation of the clay's interlayer water in surfactant aggregates. The cracks formed by passive contact with DNAPL wastes reported by Ayral et al. (2014) were up to $1 \mathrm{~mm}$ in aperture. Considering the DNAPL pool depths reported at hazardous waste sites (Oolman et al., 1995; Parker et al., 2003; Parker et al., 2004), the hydrostatic head of these pools may be greater than the entry pressure of such cracks, allowing the waste to enter.

Consequently, penetration into cracks formed by contact with DNAPL waste could be a means by which chlorinated solvents may accumulate in aquitards.

\section{Acknowledgements}

Financial support was provided by the Strategic Environmental Research and Development Program (SERDP) (Project ER-1737). Margarita Otero-Diaz was supported by a National Science Foundation (NSF) Graduate Fellowship. This paper has not been subject to review by either agency; it, therefore, does not necessarily reflect the sponsors' views, and no official endorsement should be inferred. 
Abdul, A.S., T.L. Gibson, and D.N. Rai, 1990. Laboratory studies of the flow of some organic solvents and their aqueous solution through bentonite and kaolin clays. Ground Water, 28(4):524-533.

Ayral, D., 2015. Impact of Clay-DNAPL Interactions on the Transport of Chlorinated Solvents in Low Permeability Subsurface Zones, Ph.D. dissertation, University of Michigan, Ann Arbor, MI.

Ayral, D., M. Otero, M.N. Goltz, and A.H. Demond, 2014. Impact of DNAPL contact on the structure of smectitic clay materials. Chemosphere, 95:181-186.

Ball, W.P., C. Liu, G. Xia, and D.F. Young, 1997. A diffusion-based interpretation of tetrachloroethene and trichloroethene concentration profiles in a groundwater aquitard. Water Resources Research, 33(12):2741-2757.

Boyd, S.A., M.M. Mortland, and C.T. Chiou, 1988. Sorption characteristics of organic compounds on hexadecyltrimethylammonium-smectite. Soil Science Society of America Journal, 52(3):652-657.

Brown, G., and Brindley, G., 1980. Crystal Structures of Clay Minerals and their X-Ray Identification, Mineralogical Society, London (pp. 361-410).

Brown, K.W., and J.C. Thomas, 1987. A mechanism by which organic liquids increase the hydraulic conductivity of compacted clay materials. Soil Science Society of America Journal, 51(6):1451-1459.

Burst, J.F., 1965. Subaqueously formed shrinkage cracks in clay. Journal of Sedimetnary Petrology, 35(2):348-353.

Chapman, S.W., and B.L. Parker, 2005. Plume persistence due to aquitard back diffusion following dense nonaqueous phase liquid source removal or isolation. Water Resources Research, 41, W12411, doi:10.1029/2005WR004224.

Cheng, L., P. Fenter, K.L. Nagy, and M.L. Schlegel, 2001. Molecular-scale density oscillations in water adjacent to a mica surface. Physical Review Letters, 87(15), 156103.

Del Hoyo, C., C. Dorado, M.S. Rodríguez-Cruz, and M.J. Sánchez-Martín, 2008. Physicochemical study of selected surfactant-clay mineral systems. Journal of Thermal Analysis and Calorimetry, 94(1):227-234.

Deng, Y., J.B. Dixon, and G.N. White, 2003. Intercalation and surface modification of smectite by two non-ionic surfactants. Clays and Clay Minerals, 51(2):150-161.

Dou, W., K. Omran, S.J. Grimbery, M. Denham, and S.A. Powers, 2008. Characterization of DNAPL from the U.S. DOE Savannah River site. Journal of Contaminant Hydrology, 97:75-86.

Dwarakanath, V., R.E. Jackson, and G.A. Pope, 2002. Influence of wettability on the recovery of NAPLs from alluvium. Environmental Science and Technology, 36:227-231.

Earnest, G.S., A. Beasley Spencer, S.S. Smith, W.H. Heitbrink, R.L. Mickelsen, J.D. McGothlin, and L.M. Ewers, 1997. Control of Health and Safety Hazards in Commercial Drycleaners: Chemical Exposures, Fire Hazards, and Ergonomic Risk Factors, NIOSH Publication No. 97-150, U.S. Department of Health and Human Services, Public Health Service, Centers for 
Disease Control and Prevention, National Institute for Occupational Safety and Health, Atlanta, GA.

Fendler, J.H., 1976. Interactions and reactions in reversed micellar systems. Accounts of Chemical Research, 9:153-161.

Griffin, R.A., R.E. Hughes, L.R. Follmer, C.J. Stohr, W.J. Morse, T.M. Johnson, J.K. Bartz, J.D. Steele, K. Cartwright, M.M. Killey, and P.B. DuMontelle, 1984. Migration of industrial chemicals and soil-waste interactions at Wilsonville, Illinois in Land Disposal of Hazardous Waste, Tenth Annual Research Symposium, Fort Mitchell, KY, EPA-600/9-84-007, 61-77.

Harrold, G., D.C. Gooddy, S. Reid, D.N. Lerner, and S.A. Leharne, 2003. Changes in interfacial tension of chlorinated solvents following flow through U.K. soils and shallow aquifer materials. Environmental Science and Technology, 37(9):1919-1925.

Hsu, H.-L., 2005. Determination of Interfacial Tension and Contact Angle of Dense NonAqueous Phase Liquid Waste Mixtures, Ph.D. dissertation, University of Michigan, Ann Arbor, MI.

Hsu, H.-L., and A.H. Demond, 2007. Influence of organic acid and organic base interactions on interfacial properties in NAPL-water systems. Environmental Science and Technology, 41:897-902.

Hu, Z., G. He, Y. Liu, C. Dong, X. Wu, and W. Zhao, 2013. Effects of surfactant concentration on alkyl chain arrangements in dry and swollen organic montmorillonite. Applied Clay Science, 75-76:134-140.

Imhoff, P.T., S.N. Gleyzer, J.F. McBride, L.A. Vancho, I. Okuda, and C.T. Miller, 1995. Cosolvent-enhanced remediation of residual dense nonaqueous phase liquids: Experimental investigation. Environmental Science and Technology, 29:1966-1976.

Jackson, R.E., and V. Dwarakanath, 1999. Chlorinated degreasing solvents: Physical-chemical properties affecting aquifer contamination and remediation. Ground Water Monitoring and Remediation, 19(4):102-110.

Johnston, C. T., G. Sposito, and C. Erickson, 1992. Vibrational probe studies of water interactions with montmorillonite. Clays and Clay Minerals, 40(6):722-730.

Kueper, B.H., and D.B. McWhorter, 1991. The behavior of dense, nonaqueous phase liquids in fractured clay and rock. Ground Water, 29(5):716-728.

Ladaa, T.I., C.M. Lee, J.T. Coates, and R.W. Falta, 2001. Cosolvent effects of alcohols on the Henry's Law constant and aqueous solubility of tetrachloroethylene (PCE). Chemosphere, 44(5):1137-1143.

Lee, S. Y., S.J. Kim, S.Y. Chung, and C.H. Jeong, 2004. Sorption of hydrophobic organic compounds onto organoclays. Chemosphere, 55(5):781-785.

Linn, B., and S. Stupak, 2009. Chemicals Used in Dry Cleaning Operations. Retrieved from http://www.drycleancoalition.org/chemicals/ChemicalsUsedInDrycleaning Operations.pdf.

Li, J., J.A. Smith, and A.S. Winquist, 1996. Permeability of earthen liners containing organobentonite to water and two organic liquids. Environmental Science and Technology, 30(10), 3089-3093.

Madejová, J., and Komadel, P., 2001. Baseline studies of the Clay Minerals Society Source Clays: Infrared methods. Clays and Clay Minerals, 49(5), 410-432. 
Marshall, T.J., J.W. Holmes and C.W. Rose, 1996. Soil Physics $3^{\text {rd }}$ ed., Cambridge University Press, Cambridge.

Matthieu, D.E., M.L. Brusseau, G.R. Johnson, J.L. Artiola, M.L. Bowden, and J.E. Curry, 2013. Intercalation of trichloroethene by sediment-associated clay minerals. Chemosphere, 90:459463.

McCaulou, D.R., and S.G Huling, 1999. Compatibility of bentonite and DNAPLs. Ground Water Monitoring and Remediation, 19(2):78-86.

Meunier, A., 2005. Clays. Springer, Berlin.

Moore, D.M., and Reynolds, R.C., 1989. X-ray Diffraction and the Identification and Analysis of Clay Minerals. Oxford University Press, Oxford.

Moulik, S.P., 1996. Micelles: Self-organic surfactant assemblies. Current Science, 71(5):368376.

Moulik, S. P., and K. Mukherjee, 1996. On the versatile surfactant Aerosol-OT (AOT): Its physicochemical and surface chemical behaviours and uses. Proceedings-Indian National Science Academy, Part A, 62:215-236.

Oolman, T., S.T. Godard, G.A. Pope, M. Jin and K. Kirchner, 1995. DNAPL flow behavior in a contaminated aquifer: Evaluation of field data. Groundwater Monitoring and Remediation, 15(4), 125-137.

Paradies, H.H., 1980. Shape and size of a nonionic surfactant micelle: TritonX-100 in aqueous solution. The Journal of Physical Chemistry, 84(6):599-607.

Parker, B. L., J.A. Cherry, S.W. Chapman, and M.A. Guilbeault, 2003. Review and analysis of chlorinated solvent dense nonaqueous phase liquid distributions in five sandy aquifers. Vadose Zone Journal, 2(2):116-137.

Parker, B.L., J.A. Cherry, and S.W. Chapman, 2004. Field study of TCE diffusion profiles below DNAPL to assess aquitard integrity. Journal of Contaminant Hydrology, 74(14):197-230.

Parker, B.L., S.W. Chapman, and M.A. Guilbeault, 2008. Plume persistence caused by back diffusion from thin clay layers in a sand aquifer following TCE source-zone hydraulic isolation. Journal of Contaminant Hydrology, 102:86-104.

Riddick, J., W. Bunger, and T. Sakano, 1986. Organic Solvents: Physical Properties and Methods of Purification. John Wiley, New York.

Russell, J.D., and V.C. Farmer, 1964. Infrared spectroscopic study of the dehydration of montmorillonite and saponite. Clay Minerals Bulletin, 5(32), 443-464.

Sale, T., C. Newell, H. Stroo, R. Hinchee, and P. Johnson, 2008. Frequently Asked Questions Regarding Management of Chlorinated Solvents in Soils and Groundwater. Environmental Security Technology Certification Program, Arlington, VA.

Sánchez-Martín, M.J., M.C. Dorado, C. Del Hoyo, and M.S. Rodríguez-Cruz, 2008. Influence of clay mineral structure and surfactant nature on the adsorption capacity of surfactants by clays. Journal of Hazardous Materials, 150(1):115-23.

Schwarzenbach, R.P., P.M. Gschwend, and D.M. Imboden, 2003. Environmental Organic Chemistry, Wiley-Interscience, Hoboken, NJ. 
Shen, Y.-H., 2001. Preparations of organobentonite using nonionic surfactants. Chemosphere, 44(5):989-995.

Silber, J.J., A. Biasutti, E. Abuin, and E. Lissi, 1999. Interactions of small molecules with reverse micelles. Advances in Colloid and Interface Science, 82:189-252.

Stroo, H.F., A. Leeson, J.A. Marqusee, P.C. Johnson, C.H. Ward, M.C. Kavanaugh, T.C. Sale, C.J. Newell, K.D. Pennell, C.A. Lebrón, and M. Unger, 2012. Chlorinated ethene source remediation: Lessons learned. Environmental Science and Technology, 46(12):6348-6447.

Van Oss, C.J., and R.F Giese, 1995. The hydrophilicity and hydrophobicity of clay minerals. Clays and Clay Minerals, 43(4):474-477.

Xu, Q., T.V. Vasudevan, and P. Somasundaran, 1991. Adsorption of anionic-nonionic and cationic-nonionic surfactant mixtures on kaolinite. Journal of Colloid and Interface Science, 142(2):528-534.

Zhang, W., J. Chen, B. Pan and Q. Zhang, 2005. Competitive and cooperative adsorption behaviors of phenol and aniline onto nonpolar macroreticular adsorbents. Journal of Environmental Sciences, 17(4):529-534.

Zhang, R., and P. Somasundaran, 2006. Advances in adsorption of surfactants and their mixtures at solid/solution interfaces. Advances in Colloid and Interface Science, 123-126:213-29.

Zhou, X., 1994. The Study of Restacked Single Molecular Layer Molybdenum Disulfide with Organic Tetrachloroethylene Included, B.Sc. thesis, Simon Fraser University, Burnaby, British Columbia, Canada.

Zhu, L., X. Ren, and S. Yu, 1998. Use of cetyltrimethylammonium bromide-bentonite to remove organic contaminants of varying polar character from water. Environmental Science and Technology, 32(21):3374-3378. 
Figure 1. XRD pattern of water-saturated bentonite contacted with PCE containing $3.3 \mathrm{mM}$ AOT and $3.3 \mathrm{mM}$ TritonX-100 for one week following four days of rotation.

Figure 2. FTIR spectra of water-saturated bentonite in contact with different fluids compared to the spectrum of air-dry bentonite. Wavenumbers indicated correspond to the $\mathrm{H}-\mathrm{O}-\mathrm{H}$ bending band of water.

Figure 3. Percent sorption of solutes from aqueous and PCE solutions onto Namontmorillonite. A: TritonX-100; B: AOT; C: ${ }^{13}$ C-labeled TCE. ND= below detection.

Figure 4. Schematic representation of possible arrangement of AOT and TritonX-100 in PCE in contact with water-saturated bentonite. 